\title{
ON THE FINITE LINEAR INDEPENDENCE OF LATTICE GABOR SYSTEMS
}

\author{
CIPRIAN DEMETER AND S. ZUBIN GAUTAM
}

\author{
(Communicated by Michael T. Lacey)
}

\begin{abstract}
In the restricted setting of product phase space lattices, we give an alternate proof of $\mathrm{P}$. Linnell's theorem on the finite linear independence of lattice Gabor systems in $L^{2}\left(\mathbb{R}^{d}\right)$. Our proof is based on a simple argument from the spectral theory of random Schrödinger operators; in the one-dimensional setting, we recover the full strength of Linnell's result for general lattices.
\end{abstract}

\section{INTRODUCTION}

A Gabor system is simply a collection of modulations and translations of a fixed function in $L^{2}\left(\mathbb{R}^{d}\right)$. More precisely, given any set $A \subseteq \mathbb{R}^{d} \times \mathbb{R}^{d}$ and any $f \in L^{2}\left(\mathbb{R}^{d}\right)$, the associated Gabor system is

$$
\mathcal{G}(f, A):=\left\{\mathrm{M}_{y} \mathrm{~T}_{x} f \mid(x, y) \in A \subset \mathbb{R}^{d} \times \mathbb{R}^{d}\right\},
$$

where $\mathrm{M}_{y}$ and $\mathrm{T}_{x}$ denote, respectively, the unitary operators of modulation and translation on $L^{2}\left(\mathbb{R}^{d}\right)$ given by

$$
\mathrm{M}_{y} f(t):=e^{2 \pi i y \cdot t} f(t), \mathrm{T}_{x} f(t):=f(t-x) .
$$

Here one should view $\mathbb{R}^{d} \times \mathbb{R}^{d} \cong \mathbb{R}^{d} \times \widehat{\mathbb{R}^{d}}$ as the phase space of $\mathbb{R}^{d}$; accordingly, due to the Fourier transform's intertwining of modulation and translation, one may view $\mathcal{G}(f, A)$ as the collection of "phase space translates of $f$ by $A$ " or, inspired by the case $d=1$, the collection of "time-frequency translates of $f$ by $A$ ". Most of the interest in Gabor systems stems from their "basis-like" utility in providing expansions of $L^{2}$ functions. Thus, quite naturally, a significant portion of research in the field has focused on investigating completeness and independence properties of these systems; namely, given input data $(f, A)$ in some particular class, one might like to know whether one can deduce that $\mathcal{G}(f, A)$ is an orthonormal basis, a Schauder basis, a frame, or one of a number of other basis-like objects for $L^{2}\left(\mathbb{R}^{d}\right)$ (or more generally for the span of $\mathcal{G}(f, A)$ ).

Perhaps the most basic independence property one could ask of $\mathcal{G}(f, A)$ is that it be finitely linearly independent. The Heil-Ramanathan-Topiwala Conjecture (henceforth referred to as the "HRT Conjecture") asserts that any nontrivial Gabor system should have the following property:

Received by the editors December 26, 2010 and, in revised form, September 13, 2011.

2010 Mathematics Subject Classification. Primary 42C40, 42B99, 26B99; Secondary 46B15.

Key words and phrases. Gabor systems, HRT Conjecture, random Schrödinger operators.

The first author is supported by a Sloan Research Fellowship and by NSF Grant DMS-0901208. 
Conjecture 1.1 (Heil-Ramanathan-Topiwala). Let $A \subset \mathbb{R}^{d} \times \mathbb{R}^{d}$ be a finite set and $0 \neq f \in L^{2}\left(\mathbb{R}^{d}\right)$. Then the associated Gabor system $\mathcal{G}(f, A)$ is linearly independent as a subset of $L^{2}\left(\mathbb{R}^{d}\right)$.

This conjecture was originally posed in [HRT96], in which the claim was verified under various restrictions on either the set $A$ or the function $f$ (see also Hei06. for a nice expository account); two of these results in particular point the way to the setting of this paper, viz. that of lattice Gabor systems. Namely, in the onedimensional $d=1$ setting, Heil, Ramanathan, and Topiwala proved Conjecture 1.1 under the restriction that $A$ be an arbitrary finite subset of a covolume- 1 lattice in $\mathbb{R} \times \mathbb{R}$, as well as under the alternate restriction that $A$ have cardinality at most 3 , which in turn implies that $A$ or some translate thereof is contained in a lattice in $\mathbb{R} \times \mathbb{R}=\mathbb{R}^{2}$. (We recall that a lattice in $\mathbb{R}^{n}$ is a discrete subgroup $\Gamma \leq \mathbb{R}^{n}$ of finite covolume. We define the covolume of $\Gamma$ to be the Lebesgue measure of a fundamental domain for the quotient space $\mathbb{R}^{n} / \Gamma$.)

The HRT Conjecture is of course strikingly simple in formulation; however, after the preliminary results proved in [HRT96], surprisingly little headway has been made toward its resolution. Indeed, the only landmark result of a reasonably general nature regarding this conjecture is the following 1999 theorem of Linnell, which carries the aforementioned results of [HRT96] to a natural conclusion:

Theorem 1.2 (Linnell ( $($ Lin99 $))$. Suppose $0 \neq f \in L^{2}\left(\mathbb{R}^{d}\right)$, and suppose that some translate of a finite set $A \subset \mathbb{R}^{d} \times \mathbb{R}^{d}$ is contained in a lattice of $\mathbb{R}^{d} \times \mathbb{R}^{d}$. Then the Gabor system $\mathcal{G}(f, A)$ is linearly independent in $L^{2}\left(\mathbb{R}^{d}\right)$.

Linnell's proof is based on a "twisted" version of the group von Neumann algebra techniques he developed in Lin91] to prove the (analytic) zero divisor conjecture for elementary amenable groups. While the particular von Neumann algebras exploited by Linnell arise rather naturally in Gabor analysis, there has been interest in obtaining a more ostensibly "elementary" proof of Theorem 1.2, toward a better understanding of the HRT Conjecture. An alternate proof of the one-dimensional $(d=1)$ case was recently given by Bownik and Speegle ([BS10] $)$ using the theory of shift-invariant spaces; however, as noted by the authors, the methods do not extend readily to higher dimensions.

The purpose of this paper is to provide yet another proof of Theorem 1.2 that is valid for arbitrary dimensions $d$. Unfortunately, however, our proof seems only to be able to treat the case of product lattices of the form $\Gamma \times \Lambda \leq \mathbb{R}^{d} \times \mathbb{R}^{d}$ or, more generally, lattices that can be mapped to a product lattice by a symplectic transformation of the phase space. Nonetheless, we note that this case is generic in the $d=1$ setting: any lattice in $\mathbb{R} \times \mathbb{R}$ can be symplectically mapped to a product lattice. We will discuss these issues in greater detail in Section 2 below. Our main result is the following:

Theorem 1.3. Let $\Gamma_{0}=\Gamma \times \Lambda$ be a product lattice in $\mathbb{R}^{d} \times \mathbb{R}^{d}$, where $\Gamma$ and $\Lambda$ are arbitrary lattices in $\mathbb{R}^{d}$. Suppose that some translate of a finite set $A \subset \mathbb{R}^{d} \times \mathbb{R}^{d}$ is contained in $\Gamma_{0}$, and suppose $0 \neq f \in L^{2}\left(\mathbb{R}^{d}\right)$. Then the Gabor system $\mathcal{G}(f, A)$ is linearly independent. The same result holds if $\Gamma_{0}$ is merely assumed to be a symplectic image of a product lattice; in particular, for $d=1$ the result holds for $\Gamma_{0}$ an arbitrary lattice in $\mathbb{R} \times \mathbb{R}$. 
Because of the product lattice restriction in our theorem, Linnell's proof remains the state of the art regarding the HRT Conjecture for lattices; however, our proof does have the advantage of actually yielding a slightly stronger result in this restricted setting. To wit, once one has reduced $\Gamma_{0}$ to a product lattice by a symplectic transformation, the particular structure of the modulation operators in the definition of a Gabor system becomes inconsequential; namely, one can replace the modulations with multiplication operators by any almost-everywhere-nonvanishing $L^{\infty}$ functions with a suitable periodicity 1 See Theorem 3.1 below for a precise statement of this generalization. Furthermore, one can of course consider Gabor systems $\mathcal{G}(f, A)$ whose generating functions $f$ are not elements of $L^{2}$. At the expense of sacrificing symplectic symmetry, our method of proof immediately applies to a larger class of such Gabor systems:

Theorem 1.4. Suppose $0<p \leq 2$. If some translate of $A \subset \mathbb{R}^{d} \times \mathbb{R}^{d}$ is contained in a product lattice, then the Gabor system $\mathcal{G}(f, A) \subset L^{p}\left(\mathbb{R}^{d}\right)$ is linearly independent for any $0 \neq f \in L^{p}\left(\mathbb{R}^{d}\right)$.

The methods of this paper were inspired by a connection between the HRT Conjecture and discrete Schrödinger operators that was observed by F. Nazarov and A. Volberg and pointed out to us by C. Thiele. Specifically, for parameters $\lambda>0$ and $\alpha, \theta \in \mathbb{T}=\mathbb{R} / \mathbb{Z} \cong \widehat{\mathbb{Z}}$, consider the almost Mathieu operator $\mathrm{H}_{\lambda, \alpha, \theta}$ on $\ell^{2} \mathbb{Z}$ defined by

$$
\begin{aligned}
\mathrm{H}_{\lambda, \alpha, \theta} u(n) & =u(n+1)+u(n-1)+2 \lambda \cos 2 \pi(\theta+n \alpha) \cdot u(n) \\
& =u(n+1)+u(n-1)+\left[\lambda e^{2 \pi i \theta}\right] e^{2 \pi i \alpha n} u(n)+\left[\lambda e^{-2 \pi i \theta}\right] e^{2 \pi i(-\alpha) n} u(n) .
\end{aligned}
$$

Observe that if $0 \neq u \in \ell^{2} \mathbb{Z}$ satisfies an eigenvalue equation

$$
\mathrm{H}_{\lambda, \alpha, \theta} u=E u,
$$

then one can consider the set $A=\{(-1,0),(1,0),(0, \alpha),(0,-\alpha),(0,0)\} \subset \mathbb{Z} \times \widehat{\mathbb{Z}}$ to obtain a linearly dependent analogue $\mathcal{G}(u, A)$ of a Gabor system, with the group $\mathbb{R}^{d}$ replaced by $\mathbb{Z}$. (We define $\mathcal{G}(u, A)$ in the obvious way via translations and modulations of $u$, with multiplication by characters of $\mathbb{Z}$ giving the modulations.) That is, the "Gabor system" $\mathcal{G}(u, A)$ gives a counterexample to the analogue of the HRT Conjecture over the group $\mathbb{Z}$.

The spectral theory of the almost Mathieu operator has long been a focal point in the study of discrete Schrödinger operators. For our present purposes, it suffices merely to note that for certain values of $\lambda$, $\alpha$, and $\theta$ the operator $\mathrm{H}_{\lambda, \alpha, \theta}$ has some pure-point spectrum; indeed, for $\lambda>1$ the spectrum was shown by Jitomirskaya to be entirely pure-point for almost every $\alpha, \theta \in \mathbb{T}$ (see [Jit99]). That is, one can actually find nonzero eigenfunctions $u$ and obtain counterexamples to the HRT Conjecture over $\mathbb{Z}$. On the other hand, one could recast the set $A$ above as

\footnotetext{
${ }^{1}$ Given Linnell's proof this strengthening is intuitively not so surprising, since the more general operators that arise are obviously contained in the von Neumann algebra generated by $\left\{\mathrm{M}_{y} \mathrm{~T}_{x} \mid(x, y) \in \Gamma_{0}\right\}$. However, it is not immediately clear to us whether or how easily the full strength of Theorem 3.1 below can be obtained by the methods of Lin99. In short, some of the "Ore localization" issues arising in Linnell's proof seem to become less trivial after passing from modulations to more general multiplication operators.
} 
a subset of the time-frequency lattice $\mathbb{Z} \times \alpha \mathbb{Z} \leq \mathbb{R} \times \widehat{\mathbb{R}}$ and consider a Gabor system $\mathcal{G}(f, A)$ with $0 \neq f \in L^{2}(\mathbb{R})$; this Gabor system must be linearly independent by Theorem $1.2{ }^{2}$

This contrast motivates our approach below, which essentially begins by decomposing the function $f$ along $\mathbb{Z}$-orbits $\{f(x+n)\}_{n \in \mathbb{Z}}$ and examining hypothetical linear dependence relations inside the resulting discrete Gabor systems. We show that (essentially) no such relations can occur. Indeed, in the context of the almost Mathieu operators, suppose that for fixed $\lambda$ and $\alpha$ one could find eigenfunctions $u_{\theta} \in \ell^{2} \mathbb{Z}$ of $\mathrm{H}_{\lambda, \alpha, \theta}$ satisfying (1.1) for some eigenvalue $E$ independent of almost every $\theta \in \mathbb{T}$. Then one could "piece together" the $u_{\theta}$ appropriately to obtain $f \in L^{2}(\mathbb{R})$ with $\mathcal{G}(f, A)$ violating Linnell's Theorem 1.2, so the eigenvalues $E$ must actually depend on $\theta$. In fact, the dependence must be quite "wild" in the sense that any fixed $E \in \mathbb{R}$ is $\theta$-almost surely not an eigenvalue of $\mathrm{H}_{\lambda, \alpha, \theta}$. This fact was already known prior to Linnell's result, and most of the main arguments of Section 3 below closely follow its standard proof (cf. e.g. Proposition V.2.8, CL90]). Thus, the most basic perspective from which one should approach our proof is the following: an operator from the algebra yielding a product lattice Gabor system naturally gives rise to a measurable family of operators that is rather similar to a random discrete Schrödinger operator. Moreover, the study of recurrence relations arising from a discrete translation group structure, which is central to our argument, has already yielded new results in the setting of nonlattice Gabor systems; see Dem10] and DZ12.

At this point, we should briefly discuss the common spirit of our proof for product lattices, Linnell's proof for general lattices, and the Bownik-Speegle proof of the $d=1$ case. Indeed, there is considerable aesthetic similarity between our argument and that of Bownik and Speegle, and readers familiar with the basics of von Neumann algebras will notice such a similarity among all three proofs. The core phenomenon behind all the arguments is the following: On the one hand, one uses basic generalities to obtain a priori estimates on the dimensions of certain spaces (spans of Gabor systems in the Bownik-Speegle setting, and kernels of operators in ours and Linnell's). On the other hand, one shows that a linear dependence violating the HRT Conjecture would force the appropriate dimensions to be either smaller or larger than reality permits. The current paper and BS10] use the classical notion of dimension of subspaces of $L^{2}$, while Linnell considers the Murray-von Neumann dimension of subspaces (i.e., the Murray-von Neumann trace of their associated orthogonal projections) relative to the aforementioned von Neumann algebras.

In the exact same spirit, we should also point out an elegant unpublished proof due to Thiele (Thi07]) which yields Theorem 1.2 under sufficient time-frequency decay conditions on $f$ (demanding that $f$ be Schwartz is more than sufficient).

\footnotetext{
${ }^{2}$ We must admit that this recasting is a bit artificial; $A$ is a subset of a lattice in the phase space over $\mathbb{Z}$ precisely when $\alpha$ is rational, so for $\alpha$ irrational $A$ takes on a decidedly different nature when viewed as a subset of $\mathbb{R} \times \widehat{\mathbb{R}}$. For $\alpha$ rational the methods of this paper easily yield the absence of a pure-point spectrum for $\mathrm{H}_{\lambda, \alpha, \theta}$ (in fact the spectrum is well known to be entirely absolutely continuous in that case), so $\mathrm{H}_{\lambda, \alpha, \theta}$ itself does not yield a counterexample to the HRT Conjecture over $\mathbb{Z}$. We also note that failure of the full HRT Conjecture over $\mathbb{Z}$ can be deduced by much simpler methods; indeed, taking $A^{\prime}=\{(0, \alpha),(0, \beta)\}$ for any $\alpha \neq \beta \in \widehat{\mathbb{Z}}$, the Gabor system $\mathcal{G}\left(\delta_{n}, A^{\prime}\right)$ is trivially linearly dependent for any $0 \neq n \in \mathbb{Z}$. Cf. Theorem 1 of [Kut02 for a more general discussion.
} 
Thiele's argument takes any lattice $\Gamma_{0} \leq \mathbb{R}^{d} \times \mathbb{R}^{d}$, fixes a function $f \in L^{2}\left(\mathbb{R}^{d}\right)$, and considers the Gabor systems associated to $f$ and large balls of radius $R$ in the lattice $\Gamma_{0}$. If one supposes a linear dependence in $\mathcal{G}(f, A)$ for some $A \subset \Gamma_{0}$, then the property of free abelian groups appearing in Lemma 4.2 below, which we use in a different context, shows that the spans of these Gabor systems have dimension $O\left(R^{2 d-1}\right)$. On the other hand, via an almost-orthogonality argument, the time-frequency decay assumptions on $f$ show that the dimensions must actually grow more quickly than $c R^{2 d-1+\varepsilon}$.

We conclude this introduction by pointing out one final advantage of our proof: namely, that it showcases a hierarchy of complexity in the HRT problem for lattices. Firstly, there is a jump in complexity from the one-dimensional case to that of higher dimensions, not only in the facility of metaplectic reduction (cf. Section 2) but also in obtaining a priori bounded-dimensionality conditions 3 Secondly, in the higherdimensional setting, there is likewise a jump in complexity between the product lattice setting and that of general lattices; this is most obviously illustrated by the failure of our arguments outside the product setting. Perhaps more interesting, however, is the fact that once one has "decoupled" a lattice into a product lattice in phase space (if possible), anything resembling Fourier analysis or the structure of the Heisenberg group (cf. Section 2) disappears from the picture. This is apparent in the aforementioned fact that one need not consider actual modulations in the product setting; see also Remark 5.3 of Section 5 for a further discussion in this vein.

In the following, for two quantities $A$ and $B$, we will use the notation " $A \lesssim B$ " to denote the inequality $A \leq c B$ for some constant $c$. Whenever necessary, any dependence of the implied constants $c$ on relevant parameters will be denoted by subscripts on the symbol " $\lesssim$ ".

\section{MetapleCtic ReduCtions}

A crucial observation appearing in HRT96 is that the linear independence of Gabor systems is invariant under certain affine transformations of the phase space. More precisely, suppose that for some $A \subset \mathbb{R}^{2 d} \cong \mathbb{R}^{d} \times \widehat{\mathbb{R}^{d}}$ we know that $\mathcal{G}(f, A)$ is linearly independent for all nonzero $f \in L^{2}\left(\mathbb{R}^{d}\right)$. Then we automatically know that $\mathcal{G}(f, \sigma A)$ is independent for all $f$ whenever $\sigma \in \operatorname{Sp}_{2 d}(\mathbb{R}) \ltimes \mathbb{R}^{2 d}$ is an affinesymplectic transformation; i.e. $\sigma$ is a composition of a translation and a linear transformation preserving the symplectic form on $\mathbb{R}^{2 d}$. This symmetry is essentially due to the fact that replacing $A$ by $\sigma A$ amounts to pre- and post-composing the operators $\mathrm{M}_{y} \mathrm{~T}_{x},(x, y) \in A$, by some unitary "metaplectic transformations" in $\mathcal{U}\left(L^{2}\left(\mathbb{R}^{d}\right)\right)$. This in turn is due to the fact that the linear action $\operatorname{Sp}_{2 d}(\mathbb{R}) \curvearrowright \mathbb{R}^{2 d}$ induces automorphisms of the Heisenberg group with underlying set $\mathbb{R}^{2 d} \times \mathbb{R}$, and Gabor systems in $L^{2}\left(\mathbb{R}^{d}\right)$ arise from the (unitary) Schrödinger representation of this Heisenberg group. For a more complete discussion, see e.g. Section XII.7.B of Ste93. The translation symmetry in particular accounts for the "some translate of $A$ " phrasing in Theorems 1.2 and 1.3. Henceforth we will restrict our attention to actual subsets of lattices.

\footnotetext{
${ }^{3}$ This increase in complexity is already manifest for different reasons in Linnell's argument, which can be made considerably more "concrete" for $d=1$; much of the subtlety of his proof lies in the induction step required to extend past this case. However, given that his result is often cited only for $d=1$, this feature may have been somewhat underappreciated in the literature.
} 
Accordingly, we declare two lattices $\Gamma_{1}, \Gamma_{2} \leq \mathbb{R}^{2 d}$ to be symplectically equivalent if there is some $\sigma \in \mathrm{Sp}_{2 d}(\mathbb{R})$ for which $\sigma \Gamma_{1}=\Gamma_{2}$. In the case $d=1$, the symplectic group $\mathrm{Sp}_{2}(\mathbb{R})$ luckily coincides with the entire special linear group $\mathrm{SL}_{2}(\mathbb{R})$, which is easily seen to act transitively on the space of lattices of a given covolume in $\mathbb{R}^{2}$. Thus, in particular, any lattice $\Gamma_{1} \leq \mathbb{R}^{2}$ is symplectically equivalent to a product lattice $\Gamma_{2} \leq \mathbb{R} \times \mathbb{R}$.

For $d \geq 2$, however, $\mathrm{Sp}_{2 d}$ is a proper subgroup of $\mathrm{SL}_{2 d}$, and in fact one can construct lattices in $\mathbb{R}^{2 d}$ that are not symplectically equivalent to any product lattice (cf. Remark 5.2 below). Thus, we resign ourselves to the restricted setting of product lattices, and Theorem 1.3 only treats generic lattices for $d=1$.

\section{Proof of the MAIN THEOREM}

At last, we come to the proof of Theorem 1.3. by the discussion of the previous section, we need only treat the case in which $\Gamma_{0}$ is a genuine product lattice. Our most basic perspective is identical to that of [Lin99] namely, in lieu of studying the Gabor systems $\mathcal{G}(f, A)$ themselves, we examine the algebra of operators generated by $\left\{\mathrm{M}_{y} \mathrm{~T}_{x} \mid(x, y) \in \Gamma_{0}\right\}$ in the space $\mathcal{B}\left(L^{2}\left(\mathbb{R}^{d}\right)\right)$ of bounded operators on the Hilbert space $L^{2}\left(\mathbb{R}^{d}\right)$. Indeed, suppose $f \in L^{2}\left(\mathbb{R}^{d}\right)$ is in the kernel of some operator $\mathrm{S}$ in this algebra, so that

$$
\mathrm{S} f(t)=\sum_{k=1}^{N} c_{k} \mathrm{M}_{y_{k}} \mathrm{~T}_{x_{k}} f(t)=\sum_{k=1}^{N} c_{k} e^{2 \pi i y_{k} \cdot t} f\left(t-x_{k}\right)=0
$$

for almost every $t \in \mathbb{R}^{d}$, some constants $0 \neq c_{k} \in \mathbb{C}$, and some points $\left(x_{k}, y_{k}\right) \in \Gamma_{0}$. Of course, this means precisely that the Gabor system $\mathcal{G}\left(f,\left\{\left(x_{k}, y_{k}\right)\right\}_{1 \leq k \leq N}\right)$ is linearly dependent.

In the product setting, we will deduce Theorem 1.3 (and Theorem 1.4) from the following more general result, whose proof is modeled after a basic argument from the spectral theory of random Schrödinger operators (cf. e.g. Lemma V.2.1 of CL90]).

Theorem 3.1. Suppose $\Gamma$ and $\Lambda$ are arbitrary lattices in $\mathbb{R}^{d}$. Let $\gamma_{1}, \ldots, \gamma_{N}$ be distinct elements of $\Gamma$, and let $\psi_{1}, \ldots, \psi_{N} \in L^{\infty}\left(\mathbb{R}^{d} / \Lambda\right)$ be nonzero Lebesgue-almost everywhere, viewed as $\Lambda$-periodic functions on $\mathbb{R}^{d}$. Then if $0<p \leq 2$, the operator $\mathrm{S}$ on $L^{p}\left(\mathbb{R}^{d}\right)$ defined by

$$
\mathrm{S} f(x)=\sum_{k=1}^{N} \psi_{k}(x) f\left(x+\gamma_{k}\right)
$$

has kernel $\operatorname{ker}(\mathrm{S})=\{0\}$.

Remark 3.2. Firstly, we note that the relevant phase space lattice in the context of Theorem 1.3 is $\Gamma_{0}=\Gamma \times \Lambda^{\perp}$, where $\Lambda^{\perp} \leq \widehat{\mathbb{R}^{d}} \cong \mathbb{R}^{d}$ is the annihilator or "dual lattice" of $\Lambda$. Secondly, we remark that the generalization from characters to more general $L^{\infty}\left(\mathbb{R}^{d} / \Lambda\right)$ functions is invited by the requirement that $\gamma_{1}, \ldots, \gamma_{N}$ be distinct, which is necessary for our proof. Notice that if one groups together all terms associated to a common $x_{k}$ in (3.1), one obtains a similar expression in which the $\psi_{k}$ are trigonometric polynomials (provided $\Gamma_{0}$ is a product lattice).

Proof of Theorem 3.1. For $f \in L^{p}\left(\mathbb{R}^{d}\right)$, we begin by examining $\mathrm{S} f$ along $\Gamma$-orbits; specifically, since $\Gamma \leq \mathbb{R}^{d}$ is discrete, for almost every $x \in \mathbb{R}^{d}$ we have a sequence 
$u_{x} \in \ell^{p} \Gamma$ defined by $u_{x}(\gamma)=f(x+\gamma)$. Of course, since $p \leq 2$, we have $\ell^{p} \Gamma \subseteq \ell^{2} \Gamma$; this accounts for the extension to more general $L^{p}$ spaces in Theorem 1.4. Then we have $\mathrm{S} f(x+\gamma)=\sum_{k=1}^{N} \psi_{k}(x+\gamma) u_{x}\left(\gamma+\gamma_{k}\right)$ for almost every such $x$, and accordingly for almost every $x \in \mathbb{R}^{d}$ we can study the operator $\mathrm{S}_{x} \in \mathcal{B}\left(\ell^{2} \Gamma\right)$ given by

$$
\mathrm{S}_{x} u(\gamma)=\sum_{k=1}^{N} \psi_{k}(x+\gamma) u\left(\gamma+\gamma_{k}\right)
$$

If $f \in \operatorname{ker}(\mathrm{S})$, then of course we must have $u_{x} \in \operatorname{ker}\left(\mathrm{S}_{x}\right)$ for almost every $x$. Thus, to prove the theorem it suffices to show that $\operatorname{ker}\left(\mathrm{S}_{x}\right)=\{0\} \subset \ell^{2} \Gamma$ for almost every $x \in \mathbb{R}^{d}$.

Now the key point in requiring $\Lambda$ to be a lattice is that the family of operators $\mathrm{S}_{x}$ is naturally parametrized by a finite measure space; namely, it is clear that $\mathrm{S}_{x}$ depends only on the class of $x$ in $\mathbb{R}^{d} / \Lambda$, so we can consider a Borel measurable family $x \mapsto \mathrm{S}_{x}$ from $\mathbb{R}^{d} / \Lambda$ to the space $\mathcal{B}\left(\ell^{2} \Gamma\right)$ equipped with the operator norm topology. Moreover, this family carries a natural action of the group $\Gamma$. Indeed, for any $\gamma_{0} \in \Gamma$, let $\mathrm{T}_{\gamma_{0}} \in \mathcal{U}\left(\ell^{2} \Gamma\right)$ denote the unitary translation operator given by $\mathrm{T}_{\gamma_{0}} u(\gamma)=u\left(\gamma-\gamma_{0}\right)$. Then one can readily check the commutation relation

$$
\mathrm{T}_{\gamma_{0}} \mathrm{~S}_{x} \mathrm{~T}_{\gamma_{0}}^{*}=\mathrm{S}_{x-\gamma_{0}}
$$

where the " $x-\gamma_{0}$ " should be interpreted via the obvious measure-preserving action of $\Gamma \leq \mathbb{R}^{d}$ on $\mathbb{R}^{d} / \Lambda$. Since the translations $\mathrm{T}_{\gamma_{0}}$ are unitary, this commutation relation descends to kernel projections; that is, setting $\mathrm{E}(x)$ to be the orthogonal projection onto $\operatorname{ker}\left(\mathrm{S}_{x}\right) \subset \ell^{2} \Gamma$, we have

$$
\mathrm{T}_{\gamma_{0}} \mathrm{E}(x) \mathrm{T}_{\gamma_{0}}^{*}=\mathrm{E}\left(x-\gamma_{0}\right)
$$

for almost every $x \in \mathbb{R}^{d} / \Lambda$ and all $\gamma_{0} \in \Gamma$.

Again, the goal is to show that $\operatorname{ker}\left(\mathrm{S}_{x}\right)=\{0\}$ almost surely in $x \in \mathbb{R}^{d} / \Lambda$; equivalently, we want to show that $\operatorname{tr} \mathrm{E}(x)=\operatorname{dim} \operatorname{ker}\left(\mathrm{S}_{x}\right)=0$ almost everywhere, where "tr" denotes the usual trace on $\mathcal{B}\left(\ell^{2} \Gamma\right)$. This trace function is measurable on $\mathbb{R}^{d} / \Lambda$; we defer the proof of this fact to the next section. Now for any fixed $\gamma_{0} \in \Gamma$, one has

$$
\operatorname{tr} \mathrm{E}(x)=\sum_{\gamma \in \Gamma}\left\langle\mathrm{E}(x) \delta_{\gamma}, \delta_{\gamma}\right\rangle=\sum_{\gamma \in \Gamma}\left\langle\mathrm{T}_{\gamma} \mathrm{E}(x) \mathrm{T}_{\gamma}^{*} \delta_{\gamma_{0}}, \delta_{\gamma_{0}}\right\rangle=\sum_{\gamma \in \Gamma}\left\langle\mathrm{E}(x-\gamma) \delta_{\gamma_{0}}, \delta_{\gamma_{0}}\right\rangle,
$$

where $\delta_{\gamma} \in \ell^{2} \Gamma$ denotes the characteristic function of $\{\gamma\}$. Since the translation action of $\Gamma$ preserves the natural measure on $\mathbb{R}^{d} / \Lambda$, one can eliminate it from the right-hand side by averaging:

$$
\begin{aligned}
\int_{\mathbb{R}^{d} / \Lambda} \operatorname{tr} \mathrm{E}(x) \mathrm{d} x & =\sum_{\gamma \in \Gamma} \int_{\mathbb{R}^{d} / \Lambda}\left\langle\mathrm{E}(x-\gamma) \delta_{\gamma_{0}}, \delta_{\gamma_{0}}\right\rangle \mathrm{d} x \\
& =\sum_{\gamma \in \Gamma} \int_{\mathbb{R}^{d} / \Lambda}\left\langle\mathrm{E}(x) \delta_{\gamma_{0}}, \delta_{\gamma_{0}}\right\rangle \mathrm{d} x .
\end{aligned}
$$

Now of course the summand on the right-hand side is nonnegative and independent of $\gamma \in \Gamma$, so the sum must be either zero or infinite. On the other hand, the integrand on the left-hand side is nonnegative, so if one could deduce that the sum were actually zero, one would obtain $\operatorname{tr} \mathrm{E}(x)=0$ for almost every $x \in \mathbb{R}^{d} / \Lambda$, as desired. Thus, one would like to deduce a priori that the integral on the left-hand side is finite, which could be accomplished by showing that $\operatorname{tr} \mathrm{E}(x) \lesssim 1$. 
For the case $d=1$ this bound is easily achieved as follows. By metaplectic reductions we may assume for simplicity that $\Gamma=\mathbb{Z} \leq \mathbb{R}$; thus the operators $S_{x}$ take the form

$$
\mathrm{S}_{x} u(n)=\sum_{k=1}^{N} \psi_{k}(x+n) u\left(n+m_{k}\right)
$$

for some $m_{1}<\ldots<m_{N} \in \mathbb{Z}$. Consider an arbitrary $u \in \operatorname{ker}\left(\mathrm{S}_{x}\right) \subset \ell^{2} \mathbb{Z}$. Since we assume $\psi_{k} \neq 0$ almost everywhere, we may assume that in fact $\psi_{k}(x+n) \neq 0$ for all $1 \leq k \leq N$ and all $n \in \mathbb{Z}$. Thus one obtains a recurrence relation for the values of $u$; for any $n \in \mathbb{Z}$, the equation $\mathrm{S}_{x} u(n)=0$ determines $u\left(n+m_{j}\right)$ in terms of the values $u\left(n+m_{k}\right), k \neq j$. In particular, the function $u \in \ell^{2} \mathbb{Z}$ is completely determined by its values on the interval $\left\{n \mid m_{1} \leq n \leq m_{N}\right\} \subset \mathbb{Z}$, and hence

$$
\operatorname{tr} \mathrm{E}(x)=\operatorname{dim} \operatorname{ker}\left(\mathrm{S}_{x}\right) \leq m_{N}-m_{1}+1 \lesssim 1
$$

for almost every $x$, as desired. This completes the proof of Theorem 3.1 for $d=1$.

Unfortunately, however, no such argument yields an a priori estimate $\operatorname{tr} \mathrm{E}(x) \lesssim 1$ for $d \geq 2$. This failure should be apparent from the discussion of the next section. A naïve remedy for this situation is simply to force uniform boundedness of the traces by cutting each $\mathrm{E}(x)$ with a common finite-rank projection. To this end, for $A \subset \Gamma$ let $\chi_{A}$ denote the characteristic function of $A$, and define the operator $\mathrm{E}_{A}(x):=\chi_{A} \mathrm{E}(x)$; here $\chi_{A} \in \ell^{\infty} \Gamma$ is viewed as a projection operator on $\ell^{2} \Gamma$. Again using the commutation relation (3.2), for any $\gamma_{0} \in \Gamma$ we have

$$
\operatorname{tr} \mathrm{E}_{A}(x)=\sum_{\gamma \in A}\left\langle\mathrm{E}(x) \delta_{\gamma}, \delta_{\gamma}\right\rangle=\sum_{\gamma \in A-\gamma_{0}}\left\langle\mathrm{E}(x+\gamma) \delta_{\gamma_{0}}, \delta_{\gamma_{0}}\right\rangle
$$

Just as above, we integrate over $\mathbb{R}^{d} / \Lambda$ to obtain

$$
\left|A-\gamma_{0}\right| \int_{\mathbb{R}^{d} / \Lambda}\left\langle\mathrm{E}(x) \delta_{\gamma_{0}}, \delta_{\gamma_{0}}\right\rangle \mathrm{d} x=\int_{\mathbb{R}^{d} / \Lambda} \operatorname{tr} \mathrm{E}_{A}(x) \mathrm{d} x \lesssim\left\|\operatorname{tr} \mathrm{E}_{A}\right\|_{L^{\infty}\left(\mathbb{R}^{d} / \Lambda\right)},
$$

whence

$$
\int_{\mathbb{R}^{d} / \Lambda}\left\langle\mathrm{E}(x) \delta_{\gamma_{0}}, \delta_{\gamma_{0}}\right\rangle \mathrm{d} x \lesssim \frac{\left\|\operatorname{tr} \mathrm{E}_{A}\right\|_{\infty}}{|A|}
$$

with the implied constant independent of $A$. (Here " $|A|$ " denotes the cardinality of $A \subset \Gamma$.) By the crucial "growth" property of $\Gamma$ given by Lemma 4.2 of the next section, we can find a sequence of sets $A_{n} \subset \Gamma$ such that

$$
\frac{\left|\operatorname{tr} \mathrm{E}_{A_{n}}(x)\right|}{\left|A_{n}\right|} \longrightarrow 0
$$

uniformly in almost every $x$ as $n \rightarrow \infty$. The basic idea is that while it is not immediately obvious that $\operatorname{dim} \operatorname{ker}\left(\mathrm{S}_{x}\right)<\infty$, we can still determine a large number of values of $u \in \operatorname{ker}\left(\mathrm{S}_{x}\right)$ from a relatively small number of its values. Exploiting these sets $A_{n}$ in the estimate (3.3), we obtain $\left\langle\mathrm{E}(x) \delta_{\gamma_{0}}, \delta_{\gamma_{0}}\right\rangle=0$ for almost every $x$. Since $\gamma_{0} \in \Gamma$ was arbitrary, we have

$$
\operatorname{dim} \operatorname{ker}\left(\mathrm{S}_{x}\right)=\operatorname{tr} \mathrm{E}(x)=\sum_{\gamma \in \Gamma}\left\langle\mathrm{E}(x) \delta_{\gamma}, \delta_{\gamma}\right\rangle=0
$$

for almost every $x \in \mathbb{R}^{d} / \Lambda$. Modulo the two deferred claims, this completes the proof of the theorem. 


\section{Proofs of auxiliary Results}

It remains to verify the measurability of the function $\operatorname{tr} \mathrm{E}: \mathbb{R}^{d} / \Lambda \rightarrow \mathbb{C}$ and to produce a sequence of sets $A_{n} \subset \Gamma$ satisfying the property (3.4). The first task should be viewed as a technicality and may well be standard fare. We were unable to find the specific result we require in the literature, but see for example Chapter 5 of CL90 for a discussion of related measurability issues. On the other hand, the second task seems more fundamental to Theorem 3.1. Throughout this section, we employ the same notation as that used in the proof above.

4.1. Measurability of the kernel projection trace. The idea behind proving measurability of tr $\mathrm{E}$ is simply to use functional calculus for the self-adjoint operators $\mathrm{S}_{x}^{*} \mathrm{~S}_{x}$, since $\operatorname{ker}\left(\mathrm{S}_{x}\right)=\operatorname{ker}\left(\mathrm{S}_{x}^{*} \mathrm{~S}_{x}\right)$, so that $\mathrm{E}(x)=\operatorname{proj}_{\operatorname{ker}\left(\mathrm{S}_{x}\right)}$ is a spectral projection of $\mathrm{S}_{x}^{*} \mathrm{~S}_{x}$. We begin with the following general lemma:

Lemma 4.1. Let $\mathcal{H}$ be a separable Hilbert space, and fix $u, v \in \mathcal{H}$. For an operator $\mathrm{S} \in \mathcal{B}(\mathcal{H})$, let $\mu_{\mathrm{S}}$ denote the spectral measure on $\mathbb{R}$ associated to the self-adjoint operator $\mathrm{S}^{*} \mathrm{~S}, u$, and $v$ so that

$$
\left\langle\left(\mathrm{S}^{*} \mathrm{~S}\right)^{k} u, v\right\rangle=\int_{\mathbb{R}} x^{k} \mathrm{~d} \mu_{\mathrm{S}}(x) .
$$

Then the map $\mathrm{S} \mapsto \mu_{\mathrm{S}}$ is continuous from $\mathcal{B}(\mathcal{H})$ equipped with the strong-* operator topology to the space of measures on $\mathbb{R}$ equipped with the weak-* topology.

Proof. Suppose we have a sequence $\mathrm{S}_{n} \in \mathcal{B}(\mathcal{H})$ converging to $\mathrm{S} \in \mathcal{B}(\mathcal{H})$ in the strong-* operator topology so that $\mathrm{S}_{n} \xi \rightarrow \mathrm{S} \xi$ and $\mathrm{S}_{n}^{*} \xi \rightarrow \mathrm{S}^{*} \xi$ for all $\xi \in \mathcal{H}$. By an application of the uniform boundedness principle, note that all the spectral measures $\mu_{\mathrm{S}_{n}}$ and $\mu_{\mathrm{S}}$ are supported on a common compact interval $I \subset \mathbb{R}$. Now for any polynomial $p \in \mathbb{C}[x]$, the strong-* convergence implies a fortiori that $p\left(\mathrm{~S}_{n}^{*} \mathrm{~S}_{n}\right) \rightarrow p\left(\mathrm{~S}^{*} \mathrm{~S}\right)$ in the weak operator topology on $\mathcal{B}(\mathcal{H})$; in particular, we have

$$
\int_{I} p(x) \mathrm{d} \mu_{\mathrm{S}_{n}}(x) \longrightarrow \int_{I} p(x) \mathrm{d} \mu_{\mathrm{S}}(x) .
$$

An application of the Weierstrass polynomial approximation theorem now shows that $\mu_{\mathrm{S}_{n}} \rightarrow \mu_{\mathrm{S}}$ in the weak-* topology.

With this lemma in hand, we can now check the measurability of tr E. By the definition of the operators $\mathrm{S}_{x}$ and the measurability of the functions $\psi_{k}$ appearing therein, it is clear that for each $u \in \ell^{2} \Gamma$ the quantities $\left\|\mathrm{S}_{x} u\right\|_{2}$ and $\left\|\mathrm{S}_{x}^{*} u\right\|_{2}$ are measurable functions of $x$. Thus, the map $x \mapsto \mathrm{S}_{x}$ is Borel measurable from $\mathbb{R}^{d} / \Lambda$ to $\mathcal{B}\left(\ell^{2} \Gamma\right)$ equipped with the strong-* operator topology. (It is easy to check that this implies that the map is still Borel when $\mathcal{B}\left(\ell^{2} \Gamma\right)$ is equipped with the operator norm topology, as claimed in the previous section.) Now fixing $u, v \in \ell^{2} \Gamma$ and invoking Lemma 4.1, the map $x \mapsto \int \varphi \mathrm{d} \mu_{\mathrm{S}_{x}}$ is measurable for all $\varphi \in C_{c}(\mathbb{R})$. Thus, approximating the characteristic function $\chi_{\{0\}}$ as a pointwise limit of continuous functions, so is the map

$$
x \longmapsto \mu_{\mathrm{S}_{x}}(\{0\})=\left\langle\operatorname{proj}_{\operatorname{ker}\left(\mathrm{S}_{x}\right)} u, v\right\rangle=\langle\mathrm{E}(x) u, v\rangle .
$$

In other words, the projection-valued map $\mathrm{E}$ is weakly measurable, whence it trivially follows that $\operatorname{tr} \mathrm{E}: \mathbb{R}^{d} / \Lambda \rightarrow \mathbb{C}$ is a Borel measurable function. 
4.2. Sets $A_{n} \subset \Gamma$ with property (3.4). To complete the proof of Theorem 3.1, we need to find a sequence of subsets $A_{n} \subset \Gamma$ satisfying (3.4), namely

$$
\frac{\left|\operatorname{tr} \mathrm{E}_{A_{n}}(x)\right|}{\left|A_{n}\right|} \longrightarrow 0
$$

as $n \rightarrow \infty$, uniformly in almost every $x$.

For any finite $A \subset \Gamma$, let $\mathcal{K}_{A} \subset \ell^{2} \Gamma$ denote the subspace of functions supported on $A$; thus $\mathcal{K}_{A} \cong \mathbb{C}^{|A|}$. Since $\mathrm{E}_{A}(x)=\chi_{A} \mathrm{E}(x)$, the range of $\mathrm{E}_{A}(x)$ is a subspace of $\mathcal{K}_{A}$, and the restriction $\mathrm{E}_{A}(x) \chi_{A}=\chi_{A} \mathrm{E}(x) \chi_{A}$ of $\mathrm{E}_{A}(x)$ to $\mathcal{K}_{A}$ is a self-adjoint operator of norm at most 1 ; hence

$$
\operatorname{tr} \mathrm{E}_{A}(x)=\operatorname{tr}\left(\mathrm{E}_{A}(x) \chi_{A}\right) \leq \operatorname{dim} \operatorname{ran}\left(\mathrm{E}_{A}(x) \chi_{A}\right) \leq \operatorname{dim}\left(\chi_{A} \operatorname{ker}\left(\mathrm{S}_{x}\right)\right) .
$$

Thus it suffices to find $A_{n} \subset \Gamma$ with

$$
\frac{\operatorname{dim}\left(\chi_{A_{n}} \operatorname{ker}\left(\mathrm{S}_{x}\right)\right)}{\left|A_{n}\right|} \longrightarrow 0 .
$$

To produce the sets $A_{n}$, we take the same basic perspective used to show $\operatorname{tr} \mathrm{E}(x) \lesssim 1$ in the $d=1$ setting: For any $u \in \operatorname{ker}\left(\mathrm{S}_{x}\right)$ and $\gamma \in \Gamma$, the definition of $\mathrm{S}_{x}$ allows one automatically to determine $u\left(\gamma+\gamma_{k}\right)$, provided one knows $u\left(\gamma+\gamma_{j}\right)$ for all $j \neq k, 1 \leq j \leq N \mathbb{4}$

To rephrase this perspective slightly, fix any subset $C_{0} \subset \Gamma$ and any element $\gamma_{0} \in C_{0}$. Then for any subset $C \subset \Gamma$, we define $\mathcal{P}_{C_{0}, \gamma_{0}}(C)$ to be the minimal subset of $\Gamma$ satisfying $C \subset \mathcal{P}_{C_{0}, \gamma_{0}}(C)$ and the implication

$$
\gamma+\left(C_{0} \backslash\left\{\gamma_{0}\right\}\right) \subset \mathcal{P}_{C_{0}, \gamma_{0}}(C) \Longrightarrow \gamma+\gamma_{0} \in \mathcal{P}_{C_{0}, \gamma_{0}}(C) .
$$

In other words, the larger set $\mathcal{P}_{C_{0}, \gamma_{0}}(C)$ is iteratively "grown from $C$ " by the following rule: "At any given stage, if the set contains a translate of $C_{0} \backslash\left\{\gamma_{0}\right\}$, put that same translate of $\gamma_{0}$ in the set and continue." Now by the above reasoning, if we take $C_{0}=\left\{\gamma_{1}, \ldots, \gamma_{N}\right\}$ and set $\gamma_{0}=\gamma_{k}$ for some $1 \leq k \leq N$, we see that

$$
\operatorname{dim}\left(\chi_{\mathcal{P}_{C_{0}, \gamma_{k}}(C)} \operatorname{ker}\left(\mathrm{S}_{x}\right)\right) \leq \operatorname{dim}\left(\chi_{C} \operatorname{ker}\left(\mathrm{S}_{x}\right)\right) \leq|C|
$$

for any finite $C \subset \Gamma$. The following property of $\Gamma$ will thus give the desired decay (3.4).

Lemma 4.2. Let $\Gamma \leq \mathbb{R}^{d}$ be a lattice, and fix an arbitrary finite subset $C_{0} \subset \Gamma$. Then there exist a $\gamma_{0} \in C_{0}$ and a sequence of subsets $C_{n} \subset \Gamma, n \in \mathbb{N}$, such that $\left|C_{n}\right| \lesssim n^{d-1}$ and $\left|\mathcal{P}_{C_{0}, \gamma_{0}}\left(C_{n}\right)\right| \gtrsim n^{d}$, with $\mathcal{P}_{C_{0}, \gamma_{0}}$ defined as above and the implied constants depending only on $d, \Gamma$, and $C_{0} \subset \Gamma$.

The following proof is essentially due to Thiele ([Thi07]) and is best understood geometrically; the reader may find it rather helpful to draw some pictures for the case $\Gamma=\mathbb{Z} \times \mathbb{Z} \leq \mathbb{R}^{2}$, as doing so will highlight the simplicity of the argument.

Proof. First we note that the conclusion of the lemma is clearly invariant under translations of the set $C_{0}$, possibly with the exception of the implied constants in the cardinality estimates. It will be apparent from the proof below that these constants are indeed unaffected by translations of $C_{0}$, so for simplicity we may assume $0 \in C_{0}$.

\footnotetext{
${ }^{4}$ The uniformity in $x$ of the decay follows trivially from the proof and will not be mentioned. Just as in the $d=1$ case treated above, the only implicit mention of $x$ in the argument is the requirement that $\psi_{k}(x+n) \neq 0$ for all $k$ and $n$, which as before can be guaranteed by eliminating a measure-zero set of $x$.
} 
We will choose $\gamma_{0} \in C_{0}$ to be an extreme point of the convex hull $\operatorname{ch}\left(C_{0}\right)$ of $C_{0} \subset \mathbb{R}^{d}$; again by translation-invariance of the claim, we may assume $\gamma_{0}=0 \in \mathbb{R}^{d}$. Then it is a matter of routine to check that there is a rank- $(d-1)$ subgroup $K \leq \Gamma$ with $K \cap C_{0}=\{0\}$; geometrically, one should view $K$ as the intersection of a hyperplane in $\mathbb{R}^{d}$ with the lattice $\Gamma$. The quotient $\Gamma / K$ is thus cyclic, and we can choose $x \in \Gamma$ such that $x+K$ generates $\Gamma / K$ and such that

$$
C_{0} \backslash\{0\} \subset(x+K) \cup(2 x+K) \cup \ldots \cup(m x+K)=: \bar{C}
$$

for some $m \in \mathbb{N}$. This latter set $\bar{C}$ should be viewed as a stack of hyperplanes in $\Gamma$ adjacent to $K$ that foliate a certain "strip" in the lattice $\Gamma$.

The essential observation is simply that the definition of $\mathcal{P}_{C_{0}, \gamma_{0}}$ immediately yields $\mathcal{P}_{C_{0}, 0}(\bar{C}) \supset \bar{C} \cup K$; in particular, $\mathcal{P}_{C_{0}, 0}(\bar{C})$ contains the shifted hyperplane stack $-x+\bar{C}$. By induction, we obtain

$$
\mathcal{P}_{C_{0}, 0}(\bar{C}) \supset \bigcup_{j=-\infty}^{0}(j x+K)=: H,
$$

which is the intersection of $\Gamma$ with a half-space in $\mathbb{R}^{d}$. In short, an entire half-space in $\Gamma$ can be grown from the codimension-1 "strip" $\bar{C}$ by the procedure used to define $\mathcal{P}_{C_{0}, 0}(\bar{C})$. Passing from this observation to the quantitative statement of the lemma is a simple matter, along the following lines.

Let $B_{r}(0)$ denote the usual ball of radius $r>0$ centered at 0 in $\mathbb{R}^{d}$, set $C_{n}:=$ $B_{n}(0) \cap \bar{C}$, and let $\delta$ denote the (Euclidean) diameter of $C_{0}$. Using the iterative definition of $\mathcal{P}_{C_{0}, \gamma_{0}}$, one checks that

$$
\mathcal{P}_{C_{0}, 0}\left(C_{n}\right) \supset j x+\left(B_{n+(j-1) \cdot 10 \delta}(0) \cap K\right)
$$

for each $j \in \mathbb{Z}$ satisfying $-\frac{n}{100 \delta}<j \leq 0$, say. In particular, we have

$$
\mathcal{P}_{C_{0}, 0}\left(C_{n}\right) \supset \bigcup_{-\frac{n}{200 \delta}+1 \leq j \leq-1}\left(j x+\left(B_{\frac{n}{2}}(0) \cap K\right)\right)
$$

for all sufficiently large $n$. Thus, since $K$ is a subgroup of rank $d-1$ of the lattice $\Gamma \leq \mathbb{R}^{d}$, we have $\left|C_{n}\right| \lesssim_{d, \Gamma, C_{0}} n^{d-1}$ and $\left|\mathcal{P}_{C_{0}, 0}\left(C_{n}\right)\right| \gtrsim_{d, \Gamma, C_{0}} n^{d}$, as desired.

Finally, of course, we can set $C_{0}=\left\{\gamma_{1}, \ldots, \gamma_{N}\right\}$, apply Lemma 4.2, and take $A_{n}$ to be the resulting sets $A_{n}=\mathcal{P}_{C_{0}, \gamma_{0}}\left(C_{n}\right)$ to obtain property (3.4). This completes the proof of Theorem 3.1 and hence that of Theorem 1.3 .

\section{Additional Remarks}

Remark 5.1. It is somewhat interesting to note how the proof of Theorem 3.1 degenerates for the special case of $\Lambda=\Gamma$, which in the $d=1$ setting corresponds to that of covolume- 1 phase space lattices in $\mathbb{R} \times \widehat{\mathbb{R}}$ (after exploiting metaplectic symmetries). In this case, the action of $\Gamma$ on $\mathbb{R}^{d} / \Gamma$ is of course trivial, so each $\mathrm{S}_{x}$ is simply a linear combination of translation operators on $\ell^{2} \mathbb{Z}$. One need not consider the kernel projections $\mathrm{E}(x)$ and, modulo an application of the Fourier transform, our proof becomes essentially that of Proposition 2 in [HRT96].

Remark 5.2. While the particular structure of product lattices was clearly exploited in the proof of Theorem 1.3, the difficulties posed by general lattices for our method of proof may still not be apparent. Indeed, by a rather clever combination of metaplectic transformations and passage to higher dimensions, in Lin99, Linnell 
shows that one can reduce considerations to lattices $\Gamma_{0} \leq \mathbb{R}^{d} \times \mathbb{R}^{d}$ such that $\Gamma_{0} \cap$ $\{0\} \times \mathbb{R}^{d}=\{0\} \times \mathbb{Z}^{d}$. Such lattices are temptingly close to product lattices, as their "translation components" (i.e. their projections to $\mathbb{R}^{d} \times\{0\}$ ) are lattices in $\mathbb{R}^{d}$. However, even these lattices seem in general to be out of reach for our techniques. As a particular example, in the case $d=2$, consider a lattice $\Gamma_{0} \leq \mathbb{R}^{4}$ with $\mathbb{Z}$-basis

$$
\left\{(0,0,1,0),(0,0,0,1),\left(\alpha_{1}, \alpha_{2}, \alpha_{3}, \alpha_{4}\right),\left(\beta_{1}, \beta_{2}, \beta_{3}, \beta_{4}\right)\right\},
$$

where $\alpha_{1}, \alpha_{2}, \beta_{1}, \beta_{2}$, and $\alpha_{1} \beta_{3}+\alpha_{2} \beta_{4}-\alpha_{3} \beta_{1}-\alpha_{4} \beta_{2} \in \mathbb{R}$ are linearly independent over $\mathbb{Z}$. Such a lattice is of the form to which Linnell reduces. On the other hand, by checking the values of the symplectic form on pairs of basis elements, one sees that such a $\Gamma_{0}$ cannot be symplectically equivalent to a product lattice. (A product lattice in $\mathbb{R}^{4}$ must have a $\mathbb{Z}$-basis $\left\{v_{1}, v_{2}, v_{3}, v_{4}\right\}$ with $\left[v_{1}, v_{2}\right]=\left[v_{3}, v_{4}\right]=0$, where "[., $\cdot]$ " denotes the symplectic form on $\mathbb{R}^{4}$. Routine algebra shows that our choice of $\Gamma_{0}$ has no such basis. We thank Nets Katz for pointing out this efficient method of generating examples of lattices that are not symplectically equivalent to product lattices.) Moreover, for such a $\Gamma_{0}$ with $\left(\alpha_{3}, \alpha_{4}\right)$ and $\left(\beta_{3}, \beta_{4}\right)$ generating an infinitecovolume subgroup of $\mathbb{R}^{2}$, the reader is invited to carry out a similar analysis to that in the arguments above and see what goes wrong. In short, it is not clear to us how to parametrize the operators $\mathrm{S}_{x}$ arising in the proof by a finite measure space that admits a measure-preserving action of the relevant translation lattice.

Remark 5.3. As a further comment on the nature of the product lattice setting, we note that the "post-metaplectic reduction" Theorem 3.1 can easily be reformulated for pairs of lattices in more general locally compact (not necessarily abelian) groups. The only part of the proof that causes any difficulty in extension is the analogue of Lemma 4.2 for the translation lattice; we hope to address generalizations of these results in future work. In this light, modulo metaplectic symmetries, Theorem 1.3 could be viewed as mainly being a theorem "about the translation lattice"; thus, its role as evidence for the full HRT Conjecture might be somewhat dubious.

\section{ACKNOWLEDGEMENTS}

The authors would like to thank Nets Katz and Christoph Thiele for many useful discussions regarding the HRT Conjecture. They are especially grateful to Christoph Thiele both for pointing out the connection with the almost Mathieu operator and for showing them the aforementioned proof for functions with timefrequency decay; the current work would have been impossible without either of these observations.

\section{REFERENCES}

[BS10] Marcin Bownik and Darrin Speegle, Linear independence of Parseval wavelets, Illinois J. Math. 54 (2010), no. 2, 771-785. MR2846482 (2012i:42046)

[CL90] René Carmona and Jean Lacroix, Spectral theory of random Schrödinger operators, Probability and its Applications, Birkhäuser Boston Inc., Boston, MA, 1990. MR $1102675(92 \mathrm{k}: 47143)$

[Dem10] Ciprian Demeter, Linear independence of time frequency translates for special configurations, Math. Res. Lett. 17 (2010), no. 4, 761-779. MR2661178

[DZ12] Ciprian Demeter and Alexandru Zaharescu, Proof of the HRT conjecture for $(2,2)$ configurations, J. Math. Anal. Appl. 388 (2012), no. 1, 151-159. MR.2869736

[Hei06] Christopher Heil, Linear independence of finite Gabor systems, Harmonic analysis and applications, Appl. Numer. Harmon. Anal., Birkhäuser Boston, Boston, MA, 2006, pp. 171-206. MR2249310 (2007d:42057) 
[HRT96] Christopher Heil, Jayakumar Ramanathan, and Pankaj Topiwala, Linear independence of time-frequency translates, Proc. Amer. Math. Soc. 124 (1996), no. 9, 2787-2795. MR 1327018 (96k:42039)

[Jit99] Svetlana Ya. Jitomirskaya, Metal-insulator transition for the almost Mathieu operator, Ann. of Math. (2) 150 (1999), no. 3, 1159-1175. MR.1740982 (2000k:81084)

[Kut02] Gitta Kutyniok, Linear independence of time-frequency shifts under a generalized Schrödinger representation, Arch. Math. (Basel) 78 (2002), no. 2, 135-144. MR1888415 (2003h:43005)

[Lin91] Peter A. Linnell, Zero divisors and group von Neumann algebras, Pacific J. Math. 149 (1991), no. 2, 349-363. MR.1105703 (92e:22013)

[Lin99] von Neumann algebras and linear independence of translates, Proc. Amer. Math. Soc. 127 (1999), no. 11, 3269-3277. MR1637388(2000b:46106)

[Ste93] Elias M. Stein, Harmonic analysis: real-variable methods, orthogonality, and oscillatory integrals, Princeton Mathematical Series, vol. 43, Princeton University Press, Princeton, NJ, 1993, with the assistance of Timothy S. Murphy, Monographs in Harmonic Analysis, III. MR $1232192(95 \mathrm{c}: 42002)$

[Thi07] Christoph Thiele, 2007, Personal communication.

Department of Mathematics, Indiana University, Bloomington, Indiana 47405

E-mail address: demeterc@indiana.edu

Department of Mathematics, Indiana University, Bloomington, Indiana 47405

E-mail address: sgautam@indiana.edu

Current address: School of Law, University of California, Berkeley, California 94720

E-mail address: sgautam@berkeley.edu 